\title{
Autosomal Dominant Optic Atrophy
}

National Cancer Institute

\section{Source}

National Cancer Institute. Autosomal Dominant Optic Atrophy. NCI Thesaurus. Code C84577.

An autosomal dominant hereditary condition characterized by optic atrophy and progressive visual loss. 